\title{
Glioblastoma without Remarkable Contrast Enhancement on Magnetic Resonance Imaging
}

\author{
Satoshi Utsuki, Hidehiro Oka, Yoshiteru Miyajima, Chihiro Kijima, Yoshie Yasui, Kiyotaka Fujii
}

Department of Neurosurgery, Kitasato University School of Medicine, Sagamihara, Japan.

Email: utsuki@med.kitasato-u.ac.jp

Received April 10 ${ }^{\text {th }}$ 2012; revised July $18^{\text {th }}$, 2012; accepted August $14^{\text {th }}, 2012$

\begin{abstract}
This is the report of a histological and clinical investigation of 4 cases of glioblastoma, a rare tumor, in whom poor contrast enhancement of the tumor was visualized on magnetic resonance imaging (MRI). Among the 94 patients with first-occurrence glioblastoma treated between January 2000 and August 2011, 4 were enrolled in this retrospective study. There were 2 men and 2 women, ranging in age from 41 to 70 years (mean, 57 years). All the patients underwent tumor resection, postoperative irradiation, and chemotherapy. One died of local tumor recurrence after 36 months; the remaining three remain alive as of 25 to 72 months after the initial treatment. The histopathology was glioblastoma with nuclear pleomorphism and pseudopalisading necrosis in all cases. However, the typical vascular endothelial proliferation was not found in 3 cases. All glioblastomas were immunopositive for p53 and immunonegative for epidermal growth factor receptor (EGFR) and isocitrate dehydrogenase 1 (IDH1). These glioblastomas showing unclear contrast enhancement on MRI had similar clinical and pathological characteristics, but differed in characteristics from glioblastoma patients showing marked contrast enhancement of the tumor on MRI.
\end{abstract}

Keywords: Enhancement Effect; Glioblastoma; EGFR; IDH1; p53; Secondary Glioblastoma

\section{Introduction}

Glioblastoma is characterized as a ring-enhancing mass with surrounding edema on magnetic resonance imaging (MRI) or computed tomography (CT) [1]. These tumors grow rapidly, and the reason for the ring-enhancing effect on imaging is the central necrosis in the tumor associated with the rapid growth [2,3]. Presence of a few scattered necrotic areas would be visualized as a homogeneous, but not ring enhancement of the mass on MRI and CT. However, glioblastomas have also been reported to be occasionally visualized as non-enhancing lesions on MRI [4-9]; such cases are, however, few and their clinical characteristics remain to be precisely clarified. The aim of this study was to clarify the clinical features of cases of glioblastoma, in which the tumor is visualized as a non-enhancing lesion on MRI.

\section{Materials and Methods}

\subsection{Case Selection}

From among 94 patients of intracranial glioblastoma treated between January 2000 and August 2011 at our institution, 4 were enrolled in this retrospective study. The diagnosis of glioblastoma in all of these cases was confirmed by histopathological examination, based on the World Health Organization (WHO) classification. Cases of giant cell glioblastoma and gliosarcoma were excluded. Immunohistochemical analysis was performed for p53, epidermal growth factor receptor (EGFR), Ki-67, and isocitrate dehydrogenase 1 (IDH1). PCR amplification was performed for sequencing of IDH1 and IDH2 mutations.

\subsection{Immunohistochemistry}

Sections, $4 \mu \mathrm{m}$ thick, were deparaffinized and dehydrated in xylene and a descending ethanol series. After endogenous peroxidase activity was blocked with $1 \%$ hydrogen peroxide for $10 \mathrm{~min}$ at room temperature, the sections were rinsed in tap water and antigen was retrieved in $0.01 \mathrm{M}$ sodium citrate buffer ( $\mathrm{pH}$ 6.0) containing $0.1 \%$ Tween 20 by microwaving for $10 \mathrm{~min}$. After cooling to room temperature, the sections were rinsed 3 times for 5 min each in phosphate-buffered saline (PBS, $\mathrm{pH} 7.6$ ) at room temperature. The primary antibodies were all diluted 1:100, including anti-p53 antibody (DAKO JAPAN, Kyoto, Japan), anti-EGFR antibody (sc03: Santa Cruz Biotechnologies, Santa Cruz, CA), anti-Ki-67 antibody (Ki-67: Dako Japan), and anti-IDH1 R132H antibody (H09: Dianova, Hamburg, Germany). The sections were incubated overnight at $4^{\circ} \mathrm{C}$, and then washed 3 times for 
5 min each with PBS at room temperature. The seconddary antibodies were detected by Simple Stain MAX-PO (Histofine, Nichirei Biosciences, Tokyo, Japan) for $30 \mathrm{~min}$. The sections were visualized with 3,3'-diaminobenzidine. Nuclear counterstaining was performed with Mayer's hematoxylin.

The results of the immunohistochemical stainings were scored as follows: negative (no immunopositive cells observed), slightly positive (<30\% immunopositive cells), and positive ( $>30 \%$ immunopositive cells).

\subsection{PCR Amplification and Sequencing}

The primer sequences to detect IDH1 and IDH2 mutations have been previously reported by Hartmann et al. [4]. The primers for IDH1 mutations were

5'-ACCAAATGGCACCATACGA-3' (sense) and 5'-TTCATACCTTGCTTAATGGGTGT-3' (antisense), and those for IDH2 mutations were

5'-GCTGCAGTGGGACCACTATT-3' (sense) and 5'-TGTGGCCTTGTACTGCAGAG-3' (antisense). Two microliters of the PCR amplification product was subjected to sequencing using the BigDye Terminator v3.1 Sequencing Kit (Applied Biosystems, Foster City, CA, USA). The amplification reaction was conducted over 25 cycles using $12 \mathrm{ng}$ of the sense primers for IDH1 or IDH2, each cycle comprising denaturation at $95 \mathrm{C}$ for 30 $\mathrm{s}$, annealing at $56 \mathrm{C}$ for $15 \mathrm{~s}$ and extension at $60 \mathrm{C}$ for $240 \mathrm{~s}$. Sequences were determined using the semiautomated sequencer (ABI 3730x1 Genetic Analyzer, Applied Biosystems) and the KB Basecaller Software, version 1.4 (Applied Biosystems, CA, USA).

\section{Results}

In the 4 glioblastoma patients included in this study, the tumors showed no remarkable contrast enhancement on MRI. The 4 patients comprised 2 men and 2 females, between 41 and 70 years in age (mean, 57 years). One of the 4 patients (Case 3 ) had been reported previously as a case of cerebellar glioblastoma [10]. The clinical summaries of the cases are shown in Table 1, and the results of MRI and CT are shown in Figures 1-3. Two of the 4 patients (Cases 2 and 4) showed calcification in the lesion on CT. Two of the patients (Cases 1 and 2) presented with symptoms of progressive right hemiparesis. One patient (Case 3) presented with headache and increased intracranial pressure. One patient (Case 4) developed generalized convulsions. The interval from the onset to diagnosis ranged from 1 day to 2 months. All of the patients had been treated by tumor resection via craniotomy: gross total resection of the tumor (T2-weighted image area) was achieved in 2 patients (Cases 1 and 3), while only partial resection was possible in the other 2 patients (Cases 2 and 4). All of the patients were also administered local irradiation (total radiation dose $60 \mathrm{~Gy}$ ) and concomitant chemotherapy as initial therapy. Three of the 4 patients developed local recurrence; the recurrence was diagnosed after 11 months in Case 2, and after 10 months in Cases 3 and 4 . Among the 3 cases of recurrence, one was treated with a second tumor resection (Case 3), whereas a second tumor resection was not performed in the remaining two in deference to the wishes of the patients themselves and their families (Cases 2 and 4). One of these patients (Case 2) died of local tumor recurrence 36 months after the initial treatment without even receiving second-line chemotherapy, since she developed the adverse reaction of cholecystitis following the first-line chemotherapy. One patient (Case 4) without the second tumor resection was treated with temozolomide (dose of $200 \mathrm{mg} / \mathrm{m}^{2} /$ day orally for 5 days) as the first-line chemotherapy. Upon detection of the recurrence, weekly interferon-beta treatment at $600 \times 10^{4} \mathrm{IU}$ was added, and at present, about 25 months after the initial treatment, this patient continues to receive treatment, without any increase of the tumor or deterioration of the general condition. The patient with recurrence in whom a repeat tumor excision was performed (Case 3), 32 cycles of adjuvant temozolomide therapy were administered, with the drug given orally at the dose of $200 \mathrm{mg} / \mathrm{m}^{2} /$ day for 5 days in each cycle, as second-line chemotherapy after the second tumor resection. At present, she remains disease-free without chemotherapy. One patient (Case 1) has remained alive, without evidence of recurrence, for 6 years after the initial therapy.

Table 1. Summaries of the patients with glioblastoma not showing remarkable enhancement of the lesions on magnetic resonance imaging.

\begin{tabular}{|c|c|c|c|c|c|c|c|c|c|c|c|c|c|c|}
\hline Case & Age & Sex & Location & $1 \mathrm{st}$ & $\mathrm{RT}$ & 1st CT & Recurrent & 2nd & 2nd CT & Survival & Imm & ohisto & gical fin & ings \\
\hline No & (yrs) & & & OP & (Gy) & (course) & (months) & $\mathrm{OP}$ & (course) & (months) & Ki-67 & p53 & EGFR & IDH1 \\
\hline 1 & 41 & M & Lt ... F & GTR & 60 & IAV (2) & None & None & None & Alive (73) & $14 \%$ & + & - & - \\
\hline 2 & 65 & F & $\mathrm{Lt} \ldots \mathrm{FP}$ & PR & 60 & Temo (3) & Local (11) & None & None & Dead (36) & $18 \%$ & + & - & - \\
\hline 3 & 53 & $\mathrm{~F}$ & Lt ... C & STR & 60 & IAV (2) & Local (10) & STR & Temo (32) & Alive (49) & $21 \%$ & + & - & - \\
\hline 4 & 70 & M & Rt ... TO & PR & 60 & Temo (26) & Local (10) & None & INF (78) & Alive (25) & $17 \%$ & + & - & - \\
\hline
\end{tabular}

C: cerebellum; F: frontal; FP: fronto-parietal; TO: temporo-occipital; OP: operation; PR: partial removal; STR: subtotal resection; GTR: gross total resection; CT: chemotherapy; IAV: interferon- $\beta$; ACNU, vincristin; Temo: Temozolomide; EGFR: epidermal growth factor receptor. 


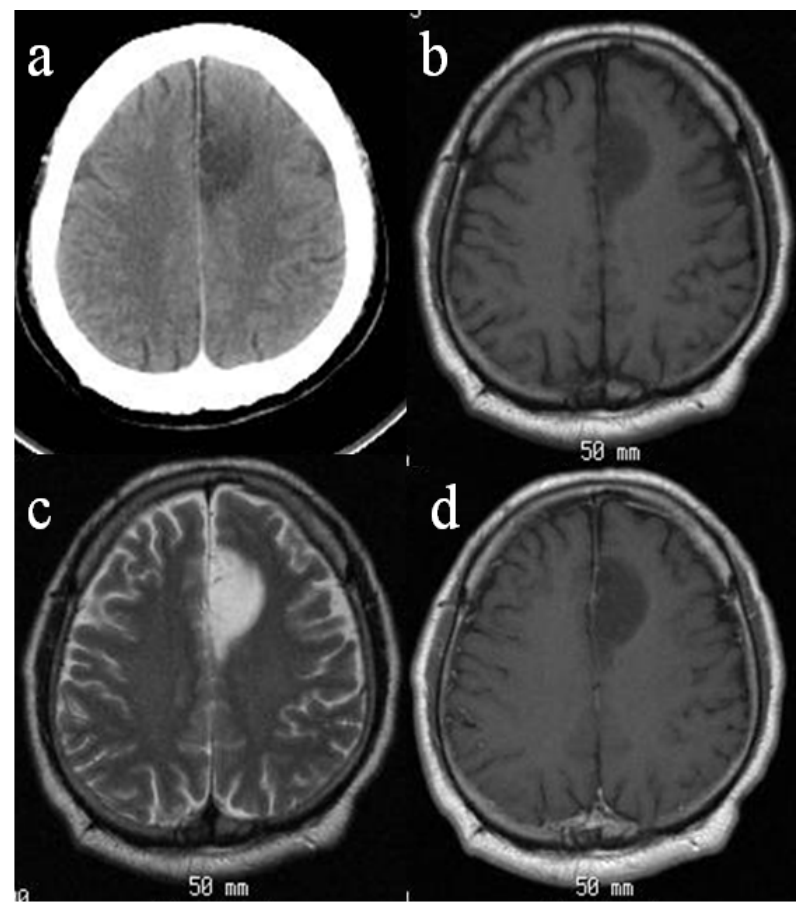

Figure 1. Computed tomography (CT) and magnetic resonance imaging (MRI) in case 1 . Axial CT showed a lowdensity mass lesion in the left frontal lobe without calcification (a). The lesion was visualized as a low intensity on T1-weighted images (WI) (b) and as a high intensity on the T2-WIs (c). The lesion showed no enhancement with gadolinium on the T1-WIs (d).

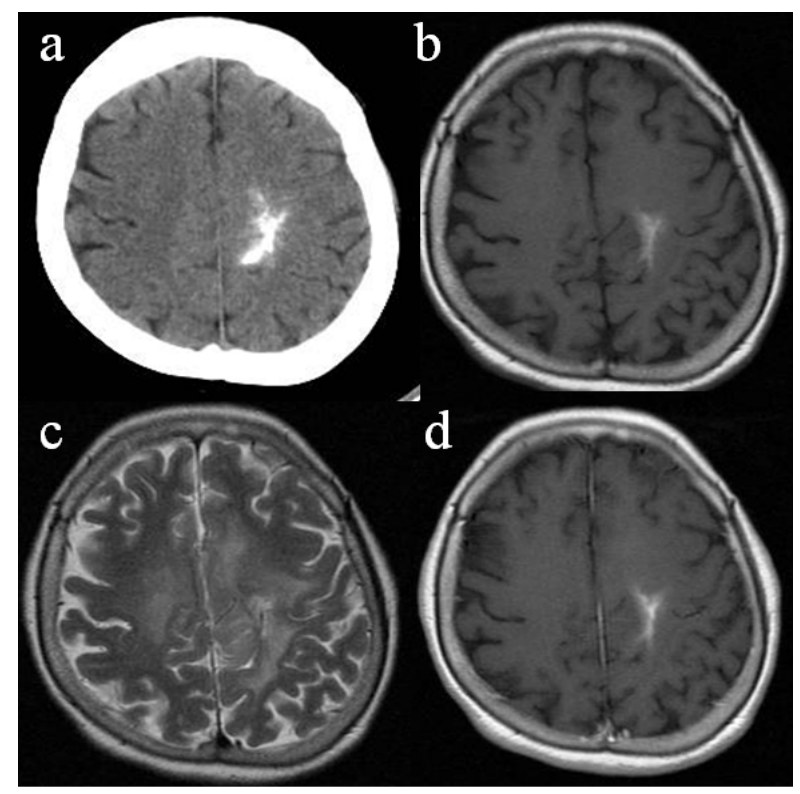

Figure 2. CT and MRI of case 2. Axial CT showed a high-density mass lesion with calcification in the left frontoparietal lobe (a). The lesion was visualized as a high or isointensity on the T1-WIs (b) and as a high intensity on the T2-WIs (c). The size of the high-intensity lesion on the T1-WIs is equal to that of the high-intensity lesions on the gadolinium on the T1-WIs (d).

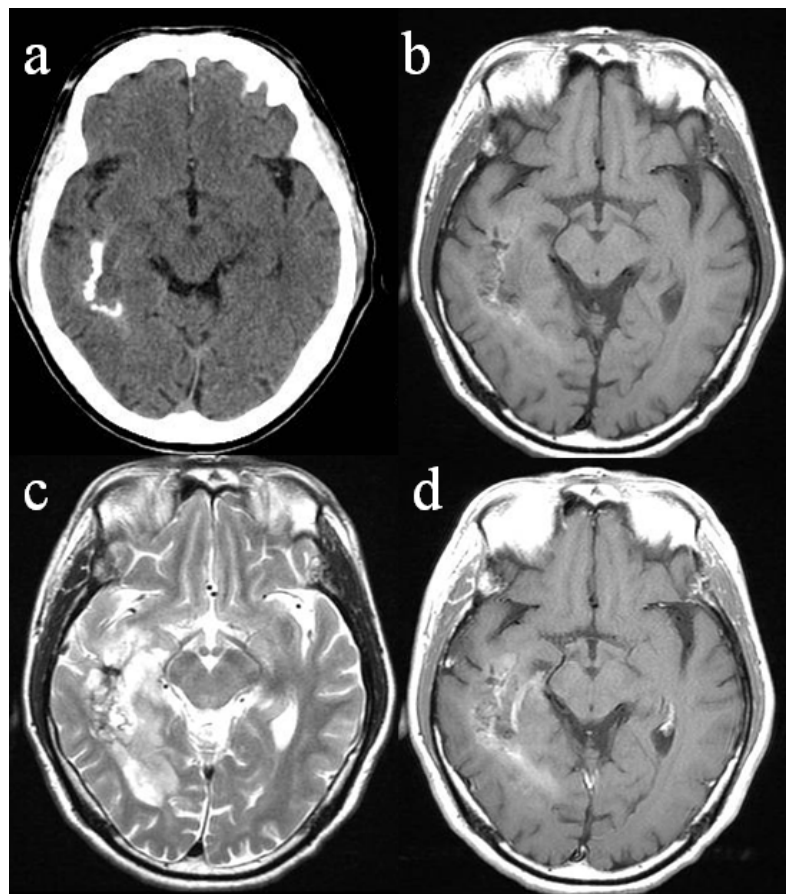

Figure 3. CT and MRI of case 4. Axial CT showed a highdensity mass lesion with calcification in the right temporooccipital lobe (a). The lesion was visualized as a mixed intensity on the T1-WIs (b) and as a high intensity on the T2-WIs (c). The lesion showed slight enhancement with gadolinium on the T1-WIs; however, the enhancement effect was not similar to the enhancement seen typically in glioblastomas (d).

Nuclear atypia, cellular pleomorphism (Figures 4(a), 5(a) and 6(a)) [10], a few mitoses and pseudopalisading necrosis (Figures 4(b), 5(b) and 6(b)) [10] were present in all of the initial tumor sections from the 4 cases. Microvascular proliferation was not found in any of the cases, while markedly expanded blood vessels were noted in all (Figures 4(c), 5(c) and 6(c)) [10]. The tumor tissues were similar throughout; however, scant lowgrade glioma histology was found in sion on CT. Two of the patients (Cases 1 and 2) pre all cases (Figures $4(\mathbf{d})$, 5(d) and 6(d)) [10]. Almost all tumor cells showed positive results of immunohistochemistry for p53 (Figures $\mathbf{4 ( e ) , 5 ( e ) ~ a n d ~ 6 ( e ) ) ~ [ 1 0 ] , ~ b u t ~ n o n e ~ o f ~ t h e ~ t u m o r ~ c e l l s ~}$ showed positive staining for EGFR (Figures 4(f), 5(f) and 6(f)) [10] or IDH1 (Figures 4(g), 5(g) and 6(g)) [10]. The Ki-67 positive rate in Cases 1, 2, 3, and 4 was 14\%, $18 \%, 21 \%$, and 17\%, respectively (Figures 4(h), 5(h) and $\mathbf{6 ( h ) )}$.

The results of PCR did not indicate the presence of any mutation of IDH1 or IDH2 in any of the 4 patients (data not shown).

\section{Discussion}

In cases where glioma is suspected on MRI as an in- 


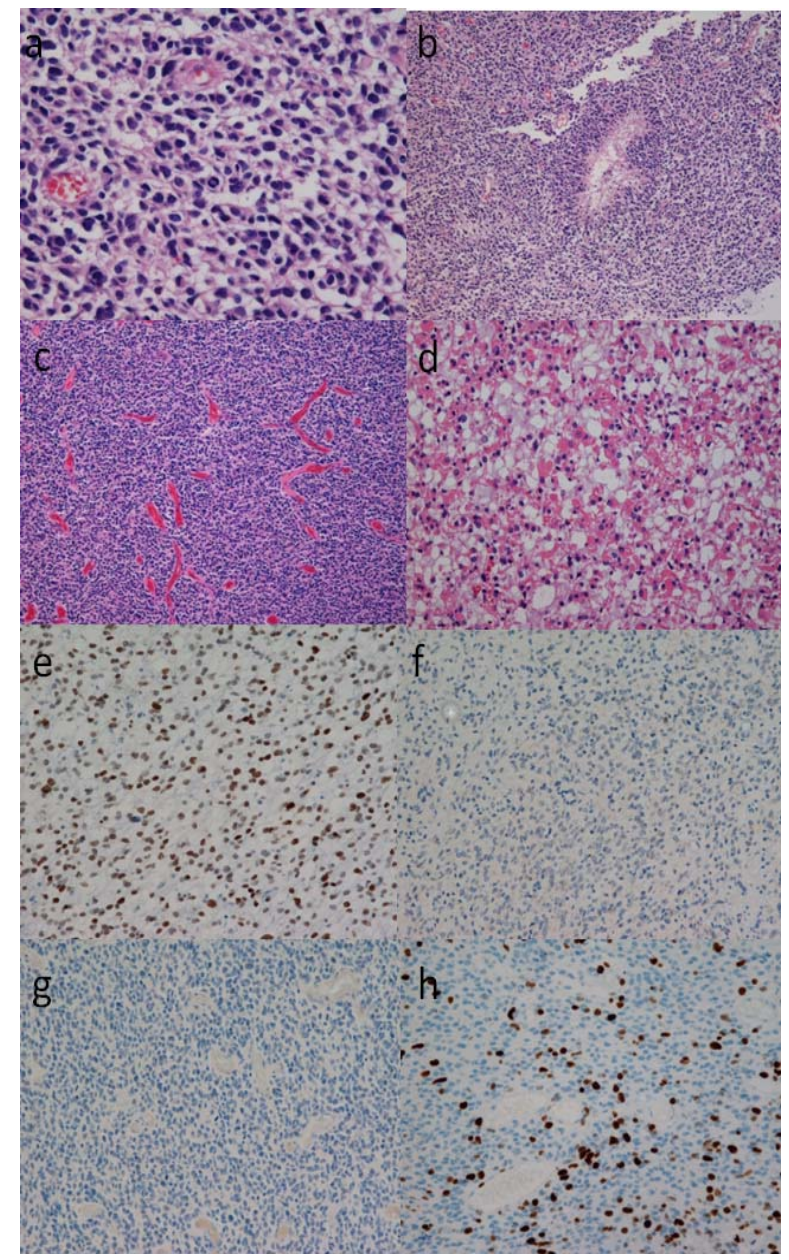

Figure 4. Photomicrographs showing the histological findings of the tumor tissue in case 1 . Tumor cells were pleomorphic with nuclear atypia and scant cytoplasm ((a), hematoxylin and eosin (HE) staining, original magnification $\times 400)$. Pseudopalisading necrosis was present ((b), HE staining, original magnification $\times 100)$. Vascular hyperplasia was marked among the tumor cells which showed a diffuse growth pattern; however, there was no evidence of microvascular proliferation ((c), HE staining, original magnification $\times 100)$. Tumor cells without nuclear atypia were present in a part of the tumor tissue. The tumor cells in this part had an abundant cytoplasm, and the cell density was low, and the cell form was clearly different from that of the tumor cells forming most parts of the tumor. Histology consistent with a low-grade glioma was limited to this area ((d), HE staining, original magnification $\times 400)$. The tumor cells were diffusely immunoreactive for p53 ((e), anti-p53 staining, original magnification $\times 100)$, while none of the cells were immunoreactive for EGFR ((f), anti-EGFR staining, original magnification $\times 100)$ or IDH1 $((g)$, anti-IDH1 staining, original magnification $\times 100)$. The Ki-67 staining index was $14 \%((g)$, anti-Ki-67 staining, original magnification $\times 100$ ).

traaxial brain lesion, but no lesion enhancement is noted after contrast injection, low-grade glioma is the most likely diagnosis, as low-grade gliomas may show vari-

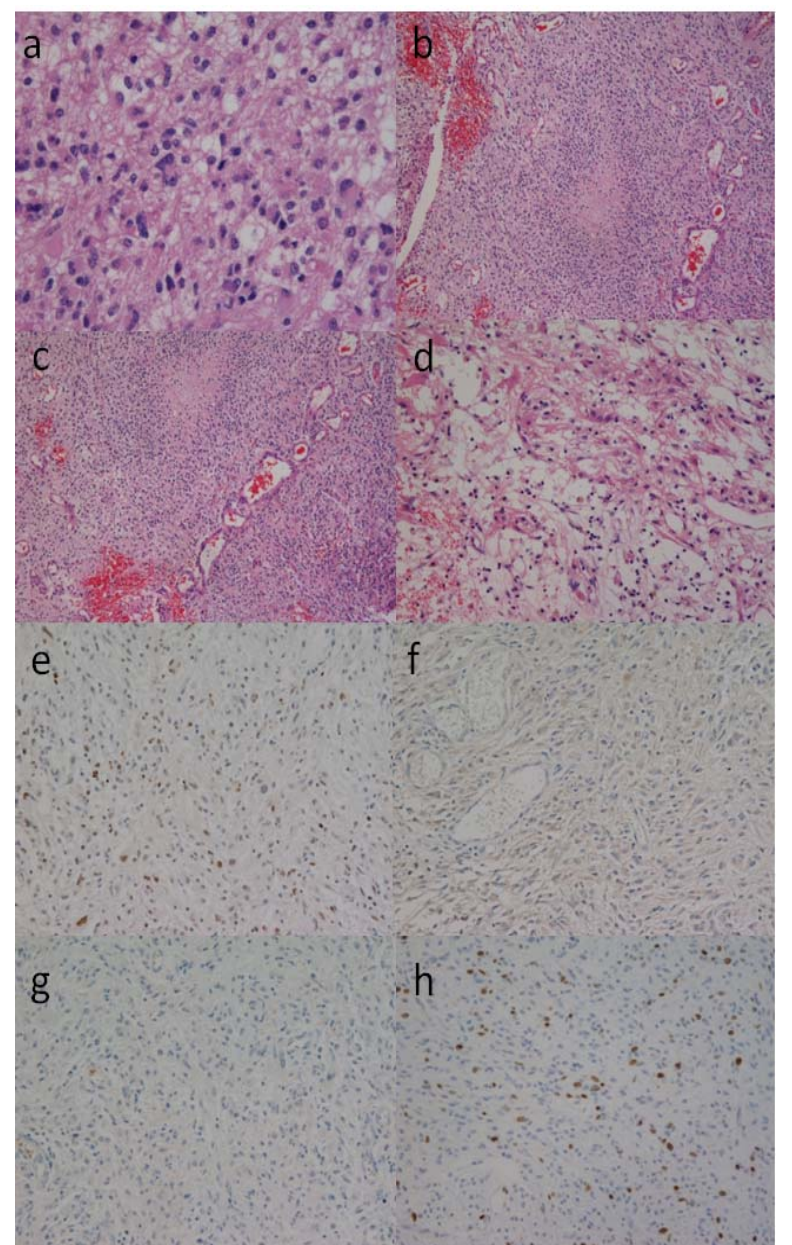

Figure 5. Photomicrographs showing histological findings of the tumor tissue in case 2. Tumor cells were pleomorphic with nuclear atypia, with some cytoplasm ((a), hematoxylin and eosin (HE) staining, original magnification $\times 400$ ). Pseudopalisading necrosis was present ((b), HE staining, original magnification $\times 100$ ). Vascular hyperplasia was marked among the tumor cells which showed a diffuse growth pattern; however, there was no evidence of microvascular proliferation ((c), HE staining, original magnification $\times 100)$. Tumor cells without nuclear atypia were present in a part of the tumor tissue. The tumor cells in this part showed many fine processes, and the cell density was low, and the cell form was clearly different from that of the tumor cells forming most parts of the tumor. Histology consistent with low-grade glioma was limited to this area ((d), HE staining, original magnification $\times 400)$. The tumor cells were diffusely immunoreactive for p53 ((e), anti-p53 staining, original magnification $\times 100)$, whereas none of the cells were immunoreactive for EGFR ((f), anti-EGFR staining, original magnification $\times 100)$ or IDH1 ((g), anti-IDH1 staining, original magnification $\times 100$ ). The Ki-67 staining index was $18 \%$ ((g), anti-Ki-67 staining, original magnification $\times 100)$.

able or no enhancement, whereas malignant or anaplastic gliomas consistently show contrast enhancement [11]. However, a few reports do indicate that occasionally higher-grade lesions may also fail to show enhancement 


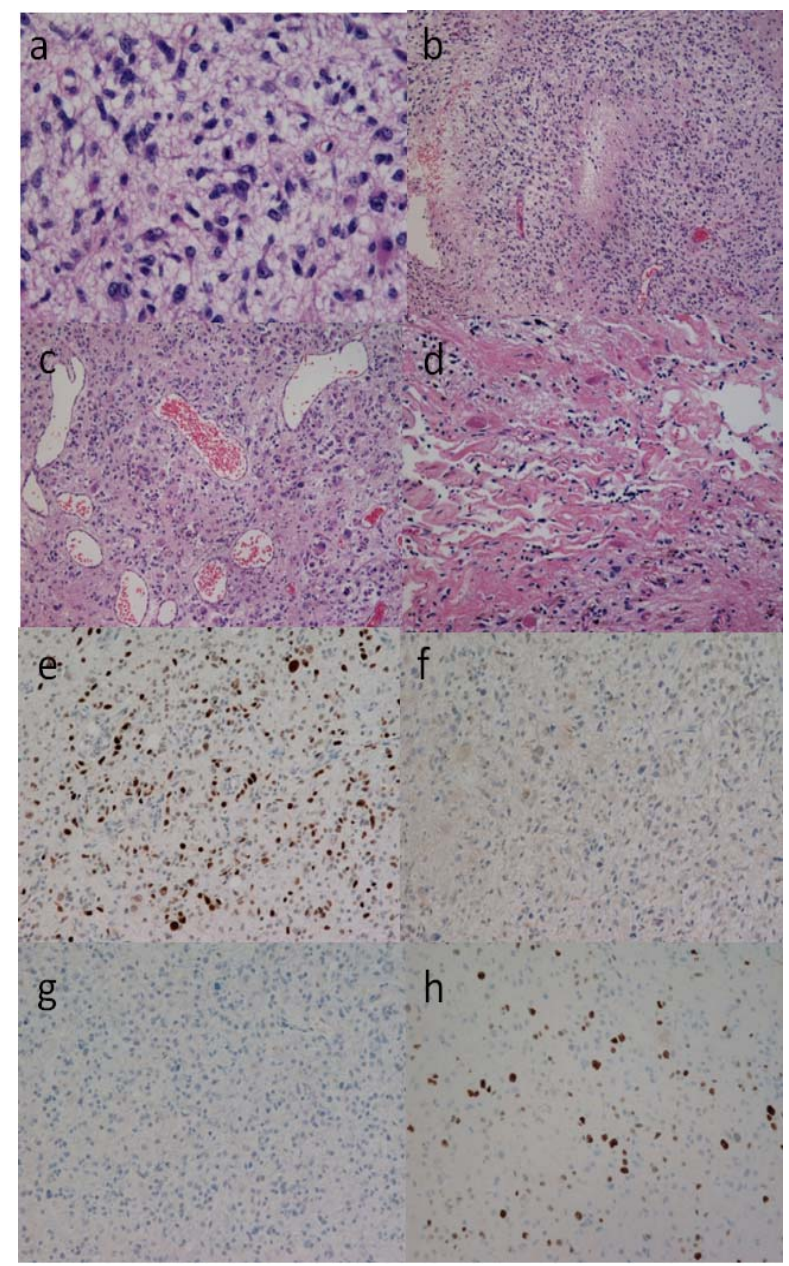

Figure 6. Photomicrographs showing the histological findings of the tumor tissue in case 4. Tumor cells were pleomorphic with nuclear atypia, and had some cytoplasm ((a), hematoxylin and eosin (HE) staining, original magnification $\times 400)$. Pseudopalisading necrosis was present $((b), \mathrm{HE}$ staining, original magnification $\times \mathbf{1 0 0})$. Vascular hyperplasia was marked among the tumor cells which showed a diffuse growth pattern; however, there was no evidence of microvascular proliferation ((c), HE staining, original magnification $\times 100)$. Tumor cells without nuclear atypia were present in a part of the tumor tissue, which also showed the presence of Rosenthal fibers. The appearance of the collagen fibers was remarkable in this part, the cell density was low, and the cell form was clearly different from that of the tumor cells forming most parts of the tumor. Histology consistent with low-grade glioma was limited to this area ((d), HE staining, original magnification $\times 400)$. The tumor cells were diffusely immunoreactive for p53 ((e), anti-p53 staining, original magnification $\times \mathbf{1 0 0})$, while none of the tumor cells were immunoreactive for EGFR ((f), anti-EGFR staining, original magnification $\times 100)$ or IDH1 $((g)$, anti-IDH1 staining, original magnification $\times 100)$. The Ki-67 staining index was $17 \%((g)$, anti-Ki-67 staining, original magnification $\times 100$ ).

on MRI [12-14]. Therefore, non-enhancement of supratentorial brain neoplasms does not exclusively indicate low-grade malignancy [12]. In an analysis of the CT findings of a series of 1988 brain tumor patients, Chamberlain et al. found no enhancement in 4 of 93 glioblastomas, 23 of 74 highly anaplastic astrocytomas, and 28 of 52 moderately anaplastic astrocytomas [15]. However, there have been no reports on the frequency of non-enhancing glioblastomas on MRI. According to a report by Pope et al., there was only one case of non-enhancing glioblastoma among 110 cases of glioblastoma examined [8]. It is known that MRI is more sensitive than CT in the detection of contrast enhancement [16]. Therefore, it is expected that the frequency of non-enhancing glioblastomas would be lower in MRI than in CT series. In this study, lack of clear contrast enhancement of the lesions on MRI was found in 4 of 94 patients with glioblastoma. A decision on the presence/absence of contrast enhancement in tumor lesions is difficult in the presence of calcification, since calcification is visualized as a high intensity on non-contrast T1-weighted images. Therefore, the judgment in respect of the presence of enhancement in our 2 cases with calcifications within the lesion was difficult. However, there was no clear enhancement effect, except in the calcified part; there was probably slight enhancement, although it was clearly different from the typical MRI findings of glioblastoma. Although the number of cases was few in this study, glioblastomas without clear enhancement on MRI may be more common than previously thought.

Enhancement of a lesion on MRI depends on the capillary basement membrane permeability of the blood brain barrier (BBB). This BBB is usually destroyed in cases of glioblastomas showing neovascularity with microvascular proliferation. Glioblastomas showing neovascularity show greater permeability than the normal BBB, because of the absence of tight junctions between the endothelial cells lining the capillary basement membrane [7]. Glioblastomas that are not associated with destruction of the BBB have been shown to exhibit poor contrast enhancement [9]. In a study where non-enhancing mass lesions suspected as low-grade neoplasms were followed up by CT or MRI without treatment, the tumors usually progressed to anaplastic astrocytoma or glioblastoma [17]. Similar findings have been reported for gliomatosis cerebri [18]. The typical MRI appearance of gliomatosis cerebri includes widespread T2-hyperintensity in the cerebral white matter without enhancement [19]. However, focal enhancement or mass effects have been reported in gliomatosis cerebri patients when malignant transformation occurs in a part of gliomatosis cerebri $[18,20]$. These regions of transformation may represent glioblastoma in their ultra-early stages of occurrence. These glioblastomas have few blood vessels so that the BBB is not destroyed; therefore, no enhancement is seen on MRI. MRI in the early stage of glioblastomas may show lack of en- 
hancement [6].

In all of the 4 patients enrolled in this study, benign gliomatous tissue was present in some parts of the tumor. If unitary hypothesis was applied, then malignant transformation of benign gliomas may be a more natural occurrence than concurrent presence of benign glioma and glioblastoma. One of the early characteristics of this pathway is the expression of p53. The timing of the transition is variable; however, most clinical studies suggest that approximately $50 \%$ of patients diagnosed as having benign gliomas will show anaplastic transformation within 5 years [21,22]. Immunohistologically, all the 4 tumors in this study were p53-positive, suggesting the involvement of p53 in the malignant transformation. Genetically, secondary glioblastomas develop more frequently in younger patients and often contain TP53 mutation, while primary glioblastomas more commonly affect elderly patients and are generally characterized by the absence of heterozygosity in $10 \mathrm{q}(\mathrm{LOH} 10 \mathrm{q})$, EGFR amplification, and TP53 mutation at a frequency of lower than 30\% [23]. More recently, it has been reported with IDH1 mutations are a highly selective molecular marker of secondary glioblastomas that complements the clinical criteria for distinguishing them from primary glioblastomas [24]. Similar results have been reported from immunohistochemical studies, and typically, secondary glioblastomas are p53-positive, EGFR-negative, and IDH1-positive, and primary glioblastomas is p53-negative, EGFR-positive, and IDH1-negative [18,19]. Cases of secondary glioblastomas with the histology of benign gliomatous lesions have been reported previously, and these tumors were also p53-positive, EGFR-negative, and IDH1-positive $[18,19]$. In all of our 4 cases, the immunohistochemical analysis revealed that the tumor was p53-positive, EGFR-negative, and IDH1-negative on immunohistochemistry, findings unlike those of either secondary glioblastomas or primary glioblastomas. These features may be characteristic of GBM [10]. Our sample size was limited, and accumulation of data from a much larger number of cases will be required in the future.

\section{Conclusion}

In suprasellar germinomas, it is rare, but significant enlargement of the optic nerve and an intact pituitary stalk can be observed at the onset. For these cases, visual impairment precedes DI, and visual impairment tends not to improve. In cases where DI precedes visual impairment without an enlargement of the optic nerve, it is very likely that treatment will improve visual impairment.

\section{REFERENCES}

[1] A. Pierallini, M. Bonamini, A. Bozzao, P. Pantano, D. D. Stefano, E. Ferone, M. Raguso, C. Bosman and L. Boz- zao, "Supratentorial Diffuse Astrocytic Tumours: Proposal of an MRI Classification," European Radiology, Vol. 7, No. 3, 1997, pp. 395-399. doi:10.1007/s003300050173

[2] L. M. DeAngelis, "Brain Tumors,” New England Journal of Medicine, Vol. 344, No. 2, 2001, pp. 114-123. doi:10.1056/NEJM200101113440207

[3] F. J. Wippold, M. Lämmle, F. Anatelli, J. Lennerz and A. Perry, "Neuropathology for the Neuroradiologist: Palisades and Pseudopalisades," American Journal of Neuroradiology, Vol. 27, No. 10, 2006, pp. 2037-2041.

[4] L. E. Bohman, K. R. Swanson, J. L. Moore, R. Rockne, C. Mandigo, T. Hankinson, M. Assanah, P. Canoll and J. N. Bruce, "Magnetic Resonance Imaging Characteristics of Glioblastoma Multiforme: Implications for Understanding Glioma Ontogeny,” Neurosurgery, Vol. 67, No. 5, 2010, pp. 1319-1328. doi:10.1227/NEU.0b013e3181f556ab

[5] R. Ramakrishna, J. Barber, G. Kennedy, A. Rizvi, R. Goodkin, R. H. Winn, G. A. Ojemann, M. S. Berger, A. M. Spence and R. C. Rostomily, "Imaging Features of Invasion and Preoperative and Postoperative Tumor Burden in Previously Untreated Glioblastoma: Correlation with Survival,” Surgical Neurology International, Vol. 1, 2010, p. 40.

[6] A. A. Cohen-Gadol, M. L. DiLuna, S. I. Bannykh, J. M. Piepmeier and D. D. Spencer, "Non-Enhancing de Novo Glioblastoma: Report of Two Cases,” Neurosurgical Review, Vol. 27, No. 4, 2004, pp. 281-285. doi:10.1007/s10143-004-0346-5

[7] J. Moore-Stovall and R. Venkatesh, "Serial Nonenhancing Magnetic Resonance Imaging Scans of High Grade Glioblastoma Multiforme," Journal of the National Medical Association, Vol. 85, No. 2, 1993, pp. 122-128.

[8] W. B. Pope, J. Sayre, A. Perlina, J. A. Villablanca, P. S. Mischel, T. F. Cloughesy, et al., "MR Imaging Correlates of Survival in Patients with High-Grade Gliomas," American Journal of Neuroradiology, Vol. 26, No. 10, 2005, pp. 2466-2474.

[9] C. Zimmer, V. Ivancevic, R. Klingebiel, K. P. Wandinger and F. K. H. van Landeghem, "Advanced Brain Tumor Imaging in Unusual Non-Enhancing Malignant Gliomas,” Klinsche Neuroradiologie, Vol. 13, No. 2, 2003, pp. 8891.

[10] S. Utsuki, H. Oka, Y. Miyajima, C. Kijima, Y. Yasui and K. Fujii, "Adult Cerebellar Glioblastoma Cases Have Different Characteristics from Supratentorial Glioblastoma," Brain Tumor Pathology, Vol. 29, No. 2, 2012, pp. 87-95.

[11] L. T. Masters and R. D. Zimmerman, "Imaging of Supratentorial Brain Tumors in Adults," Neuroimaging Clinics of North America, Vol. 3, 1993, pp. 649-687.

[12] L. E. Ginsberg, G. N. Fuller, M. Hashmi, N. E. Leeds and D. F. Schomer, "The Significance of Lack of MR Contrast Enhancement of Supratentorial Brain Tumors in Adults: Histopathological Evaluation of a Series,” Surgical Neurology, Vol. 49, No. 4, 1998, pp. 436-440. doi:10.1016/S0090-3019(97)00360-1

[13] D. Kondziolka, L. D. Lunsford and A. J. Martinez, “Unreliability of Contemporary Neurodiagnostic Imaging in 
Evaluating Suspected Adult Supratentorial (Low-Grade) Astrocytoma," Journal of Neurosurgery, Vol. 79, No. 4, 1993, pp. 533-536. doi:10.3171/jns.1993.79.4.0533

[14] M. J. Perez-Cruet, L. Adelman, M. Anderson, P. A. Roth, A. M. Ritter and S. C. Saris, "CT-Guided Stereotactic Biopsy of Nonenhancing Brain Lesions,” Stereotactic and Functional Neurosurgery, Vol. 61, No. 3, 1993, pp. 105117. doi:10.1159/000100629

[15] M. C. Chamberlain, J. A. Murovic and V. A, Levin, “Absence of Contrast Enhancement on CT Brain Scans of Patients with Supratentorial Malignant Gliomas,” Neurology, Vol. 38, No. 9, 1988, pp. 1371-1374. doi:10.1212/WNL.38.9.1371

[16] M. Castillo, "Contrast Enhancement in Primary Tumors of the Brain and Spinal Cord," Neuroimaging Clinics of North America, Vol. 4, No. 1, 1994, pp. 63-80.

[17] L. D. Recht, R. Lew and T. W. Smith, "Suspected LowGrade Glioma: Is Deferring Treatment Safe?” Annals of Neurolpgy, Vol. 31, No. 4, 1992, pp. 431-436. doi:10.1002/ana.410310413

[18] R. Nagata, K. Ikeda, Y. Nakamura, Y. Ishikawa, K. Miura, R. Sato, Y. Kawase, K. Kawabe and Y. Iwasaki, “A Case of Gliomatosis Cerebri Mimicking Limbic Encephalitis: Malignant Transformation to Glioblastoma," Internal Medicine, Vol. 49, No. 13, 2010, pp. 1307-1310. doi:10.2169/internalmedicine.49.3278

[19] G. J. Felsberg, S. A. Silver, M. T. Brown and R. D. Tien,
"Radiologic-Pathologic Correlation. Gliomatosis Cerebri,” American Journal of Neuroradiology, Vol. 15, No. 9, 1994, pp. 1745-1753.

[20] Y. M. Shin, K. H. Chang, M. H. Han, N. H. Myung, J. G. Chi, S. H. Cha and M. C. Han, "Gliomatosis Cerebri: Comparison of MR and CT Features,” American Journal of Radiology, Vol. 161, No. 4, 1993, pp. 859-862.

[21] J. Piepmeier, S. Christopher, D. Spencer, T. Byrne, J. Kim, J. P. Knisel, J. Lacy, L. Tsukerman and R. Makuch, "Variations in the Natural History and Survival of Patients with Supratentorial Lowgrade Astrocytomas,” Neurosurgery, Vol. 38, No. 5, 1996, pp. 872-879. doi:10.1097/00006123-199605000-00002

[22] D. Afra, E. Osztie, L. Sipos and D. Vitanovics, "Preoperative History and Postoperative Survival of Supratentorial Low-Grade Astrocytomas,” British Journal of Neurosurgery, Vol. 13, No. 3, 1999, pp. 299-305. doi:10.1080/02688699943727

[23] H. Ohgaki and P. Kleihues, "Genetic Pathways to Primary and Secondary Glioblastoma,” American Journal of Pathology, Vol. 170, No. 5, 2007, pp. 1445-1453. doi:10.2353/ajpath.2007.070011

[24] K. Kawarabuki, T. Ohta, N. Hashimoto, K. Wada, M. Maruno, T. Yamaki and S. Ueda, "Cerebellar Glioblastoma Genetically Defined as a Secondary One," Clinical Neuropathology, Vol. 24, No. 2, 2005, pp. 64-68. 